\title{
A Modified Model for the Lobula Giant Movement Detector and Its FPGA Implementation
}

\author{
Hongying Meng ${ }^{a}$ Kofi Appiah ${ }^{a}$ Shigang Yue ${ }^{a}$ Andrew Hunter ${ }^{a}$ \\ Mervyn Hobden ${ }^{\mathrm{b}}$ Nigel Priestley ${ }^{\mathrm{b}}$ Peter Hobden ${ }^{\mathrm{b}}$ Cy Pettit ${ }^{\mathrm{b}}$

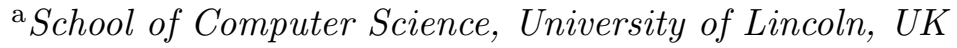 \\ ${ }^{\mathrm{b}}$ E2V Technologies PLC, Lincoln, UK.
}

\begin{abstract}
Bio-inspired vision sensors are particularly appropriate candidates for navigation of vehicles or mobile robots due to their computational simplicity, allowing compact hardware implementations with low power dissipation. The Lobula Giant Movement Detector (LGMD) is a wide-field visual neuron located in the Lobula layer of the Locust nervous system. The LGMD increases its firing rate in response to both the velocity of an approaching object and the proximity of this object. It has been found that it can respond to looming stimuli very quickly and trigger avoidance reactions. It has been successfully applied in visual collision avoidance systems for vehicles and robots. This paper introduces a modified neural model for LGMD that provides additional depth direction information for the movement. The proposed model retains the simplicity of the previous model by adding only a few new cells. It has been simplified and implemented on a Field Programmable Gate Array (FPGA), taking advantage of the inherent parallelism exhibited by the LGMD, and tested on real-time video streams. Experimental results demonstrate the effectiveness as a fast motion detector.
\end{abstract}

Key words: Neural networks, Bio-inspired vision chip, Embedded vision, Visual motion, FPGA

\section{$1 \quad 1 \quad$ Introduction}

2 For animals, such as insects, the ability to detect approaching objects is important, serving both to prevent collision as the animal moves and also to avoid capture by predators [1,2]. Evolved over millions of years, the visual collision avoidance systems in insects are both efficient and reliable. The neural circuits processing visual information in insects are relatively simple compared 
to those in the human brain and provide an appropriate model for the optical collision avoidance sensors that are needed to equip mobile intelligent machines [3].

The Lobula Giant Movement Detector (LGMD) is a wide-field visual neuron located in the Lobula layer of the Locust nervous system. The LGMD increases its firing rate in response to both the velocity of the approaching object and its proximity. It responds to looming stimuli very quickly and can trigger avoidance reactions when a rapidly approaching object is detected. It is tightly tuned to respond to objects approaching on a direct collision course [4], but produces little or no response to receding objects [5]. This makes the LGMD an ideal model to develop specialized sensors for automatic collision avoidance $[6,7]$.

A functional neural network based on the LGMD's input circuitry was developed by Rind and Bramwell [8]. This neural network showed the same selectivity as the LGMD neuron for approaching rather than receding objects and responded best to objects approaching on collision rather than near-miss trajectories. The expanding edges of colliding objects and the use of lateral inhibition were the key features of the model. This neural network has also been used to mediate collision avoidance in a real-world environment by incorporating it into the control structure of a miniature mobile robot $[9,10]$.

Inspired by the presence of direction selective neurons in the locust $[11,12]$, a new specialized translation-sensitive neural network (TSNN) has been proposed in $[13,14]$. The TSNN neuron has some common layers with the LGMD model, allowing efficiency savings in the neural computation. The TSNN fuses extracted visual motion cues from several whole-field direction selective neural networks, and is only sensitive to translational movements.

TSNN can detect the direction of translation movements very well, but it is not sensitive to movement in depth; LGMD $[8,15]$ detects the direction of movement in depth by both lateral inhibition and feed forward inhibition, where feed forward inhibition plays a critical role in inhibiting LGMD spikes to receding objects. This use of feed forward inhibition can make the system oversensitive to background movements, thus decreasing the overall sensitivity of LGMD. In this paper we propose a modified model for LGMD with several extra cells to capture the directional information for depth movements quickly, while the feed forward inhibition cell is only responsible for whole field image movements. The new model is efficiently implemented on FPGA. We have previously presented preliminary details of the new model [16], but without the full discussion or the FPGA implementation presented here.

The rest of this paper is organized as follows: In section 2, we give an overview of related work. In section 3, we address the modified LGMD model and its 
software simulation. In section 4, we discuss the FPGA design and present experimental results from the hardware implementation; in section 5 we present conclusions.

\section{Related work}

Motion sensors are presently employed in a wide variety of applications including surveillance, aerospace and automotive safety control systems and navigational systems. Motion sensors are primarily based on ultrasound, passive infrared (PIR) and radar detectors. Ultrasonic motion sensors are commonly used for automatic door openers and security alarms. PIR sensors are perhaps the most frequently used home security sensor. Radar sensors use microwave signals and detect intrusion by comparing a transmitted signal with a received echo signal and detect a Doppler shifted echo.

Recent years, vision sensors [17] are becoming increasingly cheap and reliable, and may potentially be used for a number of tasks, including collision avoidance, navigation and object recognition. This makes it desirable to develop efficient collision avoidance algorithms using visual sensors. However, collision avoidance is computationally demanding, and requires a very quick response from the sensor [18-20].

Motion patterns in 2D video imagery contain distance information about objects in a 3D environment [21]. An object on a collision course with the sensor system displays movement in depth. There is a substantial body of literature on detection of depth from vision, primarily using stereo vision [22-24], although there is also some interesting work using monocular vision [25-27]. A looming object (one moving towards the sensor) appears to expand, which suggests using optic flow algorithms and looking for a divergent flow pattern. A number of authors have suggested using optic flow to compute obstacle timeto-collision from a moving robot [28-30,26,31]. However, optic flow algorithms are computationally expensive, and the difficulty in estimating accurate optic flow from real world data [32] make these insufficiently robust for general applications. Alternatively some collision avoidance systems are based on the fusion of vision and radar sensors [33], exploiting the advantages of each.

Bio-inspired vision algorithms are a particularly good candidate for collision avoidance systems as they use simple, easily parallelized algorithms. Galbraith et al [34] proposed a population coded algorithm, built on established models of motion processing in the primate visual system, to estimate the time-tocollision with improved performance over the optic flow based method. However, it remains computationally expensive. 
There have been a number of attempts to design a bio-inspired neural chip based on the LGMD neural network for motion detection. This bio-inspired neural model features a particularly simple and highly parallelizable architecture, which may consequently be efficiently implemented on hardware, leading to low cost and low power dissipation. It provides a much quicker response that the normal monocular or stereo visual sensors.

Laviana et al [35] proposed a vision chip architecture based on the LGMD model described in [36] - a simplification of the model proposed in [8,1]. The system includes an FPGA, a block of $100 \times 150$ 6-bit retinotopic units, a controller, a 16Kbits SRAM memory block, I/O registers and some other peripherals needed for addressing, timing control, digital-to analog converters and temperature monitoring. The FPGA chip uses $0.35 \mu \mathrm{m} 2 \mathrm{P}-2 \mathrm{M}$ technology. Okuno and Yagi [37,38] implemented an LGMD model based on [8], for a real-time collision avoidance vision sensor. The system consists of an analog VLSI silicon retina and a digital FPGA circuit. The system responds selectively to colliding objects even in complicated real-world situations. These two implementations both use FPGA, but have some important limitations: first, they are based on the original LGMD model, which lacks movement direction information; second, both have built-in restrictions due to their tight integration with the non-FPGA parts of the system (e.g. the retinotopic units), and therefore are not general purpose FPGA implementations.

In this paper, in order to reduce the false alarm caused by receding objects in the LGMD model, we modify the model to distinguish approaching movement from receding movement. The modified model retains simplicity in the software and hardware implementation. Its resource usage is low enough to admit integration with other functions on the FPGA, and it can be transferred to any FPGA development platform. This design can achieve a very high frame rate and can be applied in real-time vehicular collision avoidance systems with a low false alarm rate.

\section{Modified LGMD neural network model}

The LGMD based neural network proposed in this paper is based on previous studies described in $[8,10,39,40]$. The modified neural network is shown in figure 1. The LGMD neural network in [8-10] was composed of four groups of cells - photoreceptor cells $(P)$; excitatory and inhibitory cells ( $E$ and $I)$; summing cells $(S)$; and two single cells for feed-forward inhibition $(F F I)$ and LGMD. The model in $[40,15]$ has an extra set of grouping cells between the summing cells and LGMD. This allows clusters of excitation in the summary cells to feed into the LGMD cell, which is useful for collision detection in complex backgrounds. 
The input to the $P$ cells is the luminance change. Lateral inhibition is indicated with dotted lines and has a one frame delay. Excitation is indicated with black lines and has no delay. The FFI also has a one frame delay. The input to FFI is the luminance change from the photoreceptor cells. The problem of parameter selection in this LGMD model has been tackled in [41].

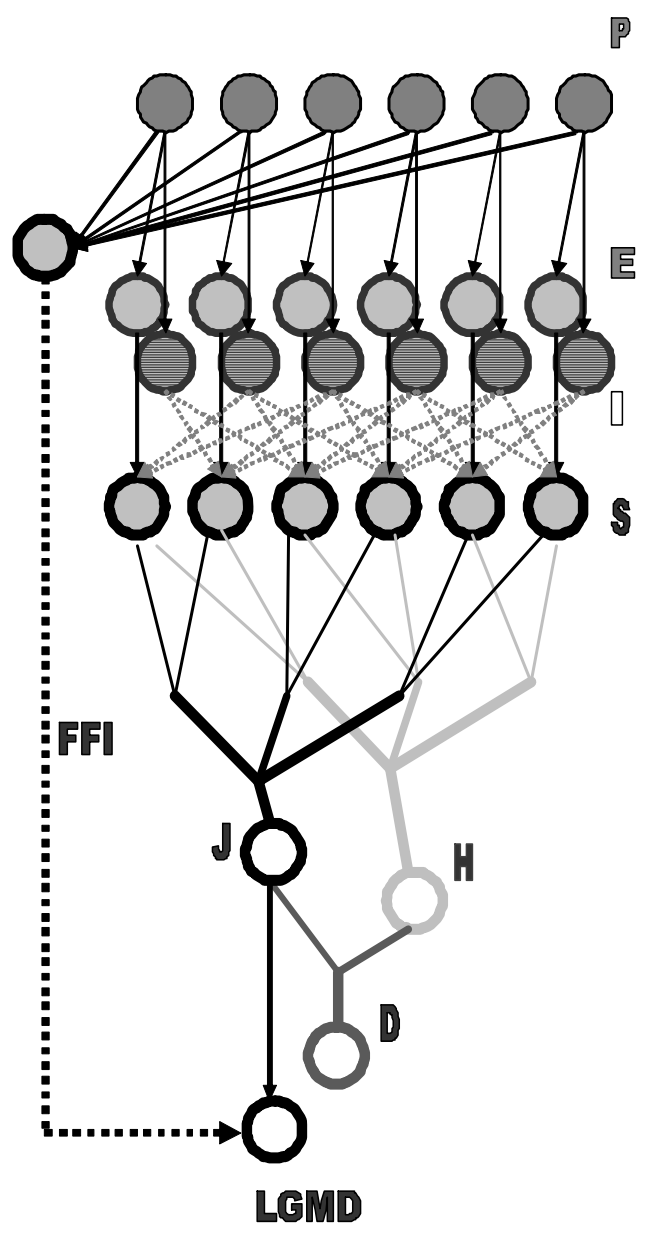

Fig. 1. A schematic illustration of the modified LGMD neural network model. There are four groups of cells and five single cells: photoreceptor cells (P); excitatory and inhibitory cells (E and I); summing cells (S); grouping cells ( $\mathrm{J}$ and $\mathrm{H})$; depth movement direction cell (D); the LGMD cell and the feed forward inhibition cell (FFI).

The model in [40] works very well for collision detection in complex environments. However, it cannot distinguish the direction of moving objects in depth. For example, it will respond to both an approaching object and a receding object with high excitation level, especially when an object is very close. To enhance the ability to recognize the direction of the moving object in depth, we add a new neural layer with two grouping cells $J$ and $H$, and a 
new cell $D$ to give in-depth direction information; see figure 1 . Note that the $J, H$ and $D$ cells may not have exact counterparts in the locust visual system. The model is described in detail below.

\subsubsection{P layer}

The first layer contains the photoreceptor $P$ cells arranged in a retinotopic matrix; the input frame pixel luminance $L_{f}$ is captured by each photoreceptor cell. The cells calculate the luminance change, which forms the output of this layer, using the equation:

$$
P_{f}(x, y)=\sum_{i}^{n_{p}} p_{i} P_{f-i}(x, y)+\left(L_{f}(x, y)-L_{f-1}(x, y)\right)
$$

where $P_{f}(x, y)$ is the change of luminance corresponding to pixel $(x, y)$ at frame $f, x$ and $y$ are the index into the matrix, $L_{f}$ and $L_{f-1}$ are the luminance, subscript $f$ denotes the current frame and $f-1$ denotes the previous frame, $n_{p}$ defines the maximum number of frames (or time steps) the persistence of the luminance change can last, the persistence coefficient $p_{i} \in(0,1)$ and

$$
p_{i}=\left(1+e^{\mu i}\right)^{-1}
$$

where $\mu \in(-\infty,+\infty)$ and $i$ indicates the previous $i^{\text {th }}$ frame counted from the current frame $f$. The LGMD neural network detects potential collision by responding to expansion of the image edges, a strategy that does not rely on object appearance. If there is no difference between successive images, the $P$ cells are not excited.

\subsubsection{E layer}

The output of the $P$ cells forms the inputs to two separate cell types in the next layer. The excitatory cells pass excitation directly to their retinotopic counterparts in the third layer, the $S$ layer. The excitation $E(x, y)$ in an $E$ cell has the same value as that in the corresponding $P$ cell. The lateral inhibition cells pass inhibition, after 1 image frame delay, to their retinotopic counterpart's neighboring cells in the $S$ layer. The inhibition strength of a cell in this layer is given by:

$$
I_{f}(x, y)=\sum_{i} \sum_{j} P_{f-1}(x+i, y+j) w_{I}(i, j),(i f i=j, j \neq 0)
$$

where $I_{f}(x, y)$ is the inhibition corresponding to pixel $(x, y)$ at current frame $f, w_{I}(i, j)$ is the local inhibition weight. Note that $i$ and $j$ are not allowed 
to be equal to zero simultaneously. Consequently, inhibition spreads out to neighboring cells in next layer rather than to the direct counterpart.

In our experiments, on both software simulation and hardware implementation, the local inhibition weight $w_{I}(i, j)$ are set to 0.25 for the four nearest neighbors and 0.125 for the four diagonal neighbors. These values are especially convenient for hardware implementation.

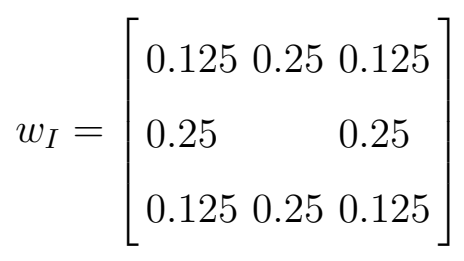

\subsubsection{S layer}

The excitatory flow from the $E$ cells and inhibition from the $I$ cells is summed by the $S$ cells using the following equation:

$$
S_{f}(x, y)=E_{f}(x, y)-I_{f}(x, y) W_{I}
$$

where $W_{I}$ is the inhibition weight (usually less than 0.8 ; 0.35 was empirically chosen in our experiments). Excitations that exceed a threshold value are able to reach the summation cell LGMD:

$$
\tilde{S}_{f}(x, y)= \begin{cases}S_{f}(x, y), & \text { if } S_{f}(x, y) \geq T_{r} \\ 0, & \text { if } S_{f}(x, y)<T_{r}\end{cases}
$$

where $T_{r}$ is the threshold.

\subsubsection{J H cells}

The $J$ and $H$ cells are the two new grouping cells for depth movement direction recognition. The $J$ cell is exactly the same as the LGMD cell in the previous LGMD model in terms of spatiotemporal structure and the value it holds: it sums the $S$ cell activations to give an overall network response. The $H$ cell shares the same structure as $J$ cell, but with a temporal difference, having a one frame delay from $J$.

$$
\begin{aligned}
& J_{f}=\sum_{x, y} \tilde{S}_{f}(x, y) \\
& H_{f}=J_{f-1}
\end{aligned}
$$


(a)

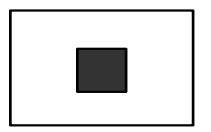

(b)

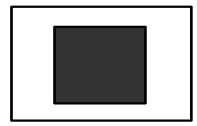

$L_{i-1}$

$$
D_{f}=a b s\left(J_{f}\right)-a b s\left(H_{f}\right)
$$

$\tilde{D}_{f}= \begin{cases}1, & \text { if } D_{f} \geq T_{D} \\ 0, & \text { if }-T_{D}<D_{f}<T_{D} \\ -1, & \text { if } D_{f} \leq-T_{D}\end{cases}$

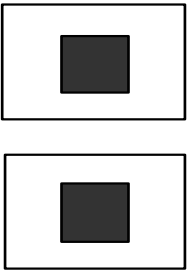

$L_{i}$
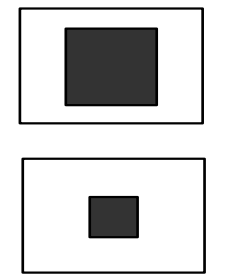

$L_{i+1}$
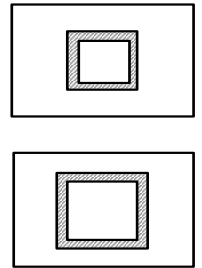

$A_{i}$
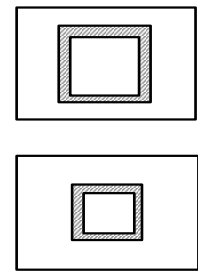

$A_{i+1}$

Fig. 2. An illustration of the difference between approaching (a) and receding (b) depth movement. $L_{i-1}, L_{i}$ and $L_{i+1}$ are three consecutive three frames in the video clip. $A_{i}$ and $A_{i+1}$ are the affected areas while doing the frame subtractions between these frames. In the approaching case, the affected area gets larger; in the receding case smaller.

The $D$ cell estimates the direction of movement in depth very well. It exploits the property that a looming object gets larger, whereas a receding one gets smaller; see figure 2. Due to the aperture effect, a moving object may only cause detectable changes around the edge (or internal contrast boundaries); however, at constant speed the size of the area of change is still related to the direction of movement in depth. When an object is moving away, abs $\left(J_{f}\right)$ is smaller than abs $\left(H_{f}\right)$. When an object is approaching, abs $\left(J_{f}\right)$ is bigger than $a b s\left(H_{f}\right)$. The absolute value function on $J$ and $H$ cells is used to cancel the different effects on their values when the object is darker or brighter than the background. In order to distinguish slow movements we add a threshold $T_{D}$ for $D_{f}$. We then get a simple variable $\tilde{D}$ that has only three values: ' 0 ', ' 1 ' and ' -1 ', where ' 1 ' stands for approaching, ' -1 ' for receding and ' 0 ' for no significant movement. The threshold $T_{D}$ depends mainly on the size of the image. 
When augmented with the above cells, the LGMD model recognizes directional information for depth movements quickly. The feed forward inhibition cell, as detailed later, is able to concentrate on whole image movements to avoid perturbation from background movements.

\subsubsection{LGMD cell}

The membrane potential $J$ is then transformed to a spiking output using a sigmoid transformation,

$$
L G M D_{f}=\left(1+e^{-J_{f} n_{c e l l}^{-1}}\right)^{-1}
$$

where $n_{\text {cell }}$ is the total number of the cells in $S$ layer. Since $J_{f}$ is greater than or equal to zero (as equation 7 is a sum of absolute value), the sigmoid membrane potential $L G M D_{f}$ varies from 0.5 to 1 . The collision alarm is decided by the spiking of cell LGMD. If the membrane potential $L G M D_{f}$ exceeds the threshold $T s$, a spike is produced. A certain number of successive spikes, denoted by $S_{L G M D}$, will trigger the collision alarm in the LGMD cell. Of course, in the modified model, the collision alarm is only triggered under the condition that $\tilde{D}=1$ where the moving object is approaching. The spikes may be suppressed by the FFI cell when whole field movement occurs [39].

\subsubsection{FFI cell}

If it is not suppressed during turning, the network may produce spikes and even false collision alerts due to sudden changes in the scene. The feed forward inhibition and lateral inhibition work together to cope with such whole field movement [39]. The FFI excitation at the current frame is gathered from the photoreceptor cells with one frame delay,

$$
F_{f}=\sum_{j}^{n_{a}} \alpha_{f-j}^{F} F_{f-j}+\sum_{x=1}^{n_{r}} \sum_{y=1}^{n_{c}} a b s\left(P_{f-1}(x, y)\right) n_{c e l l}^{-1}
$$

where $\alpha_{f-j}^{F}$ is the persistence coefficient for $F F I$ and $\alpha_{f-j}^{F} \in(0,1), n_{a}$ defines how many time steps the persistence can last.

Once $F_{f}$ exceeds its threshold $T_{F F I}$, spikes in the LGMD are inhibited immediately. The threshold $T_{F F I}$ is also adaptable,

$$
T_{F F I}=T_{F O}+\alpha_{f f i} T_{F F I_{f-1}}
$$


where $T_{F O}$ is the initial value of the $T_{F F I}$, the adaptable threshold is decided by the previous $T_{F F I}$ and $\alpha_{f f i}$ is a coefficient. The parameters, including $T_{F O}$, $\alpha_{f f i}$, are tuned to the application, the value depending on the image size and the style of camera movement. In the case when the camera is nearly stable, the FFI cell is normally ignored as it rarely reacts.

\subsection{Simulation results on the proposed model}

Two data sets were used to test the efficiency and stability of the proposed LGMD model in software simulation. The first experiment is on a simulated data set that demonstrates carefully-calibrated approaching and receding movements. The second data sets are two recorded video clips. The parameters were kept the same in all experiments; values are shown in table X. [YOU BEST REPLACE THIS X!]. The simulation was performed using MATLAB. Because the camera was still in the following experiments, FFI cell was ignored. Other parameters used in the all following experiments are listed in the table 1

Table 1

Settings for the control parameters of the LGMD model where $n_{r}$ and $n_{c}$ are the numbers of the pixels in the horizontal and vertical directions in the video frame.

\begin{tabular}{|c|c|c|c|c|c|c|}
\hline$n_{p}$ & $\mu$ & $p_{1}$ & $W_{I}$ & $T_{r}$ & $T_{D}$ & $n_{\text {cell }}$ \\
\hline \hline 1 & 1.95 & 0.125 & 0.25 & 3 & $0.25^{*} n_{r} * n_{c}$ & $n_{r} * n_{c}$ \\
\hline
\end{tabular}

\subsubsection{Results on simulated data set}

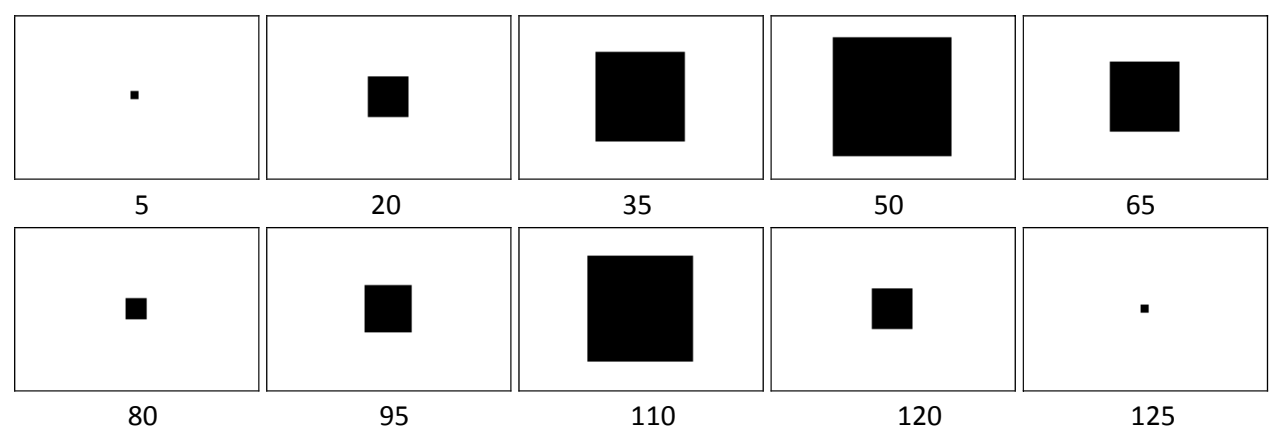

Fig. 3. Selected frames from the simulated sequence. The square object looms and recedes twice, with the second sequence at twice the speed of the first.

We created a sequence containing 125 frames, resolution $150 \times 100$, of a square black object on a white background. The object alternatively approaches and recedes. Sample frames are shown in figure 3. Initially the square is stationary with size $3 \times 3$. It looms from frame $5-41$, then recedes from frame $41-79$, 


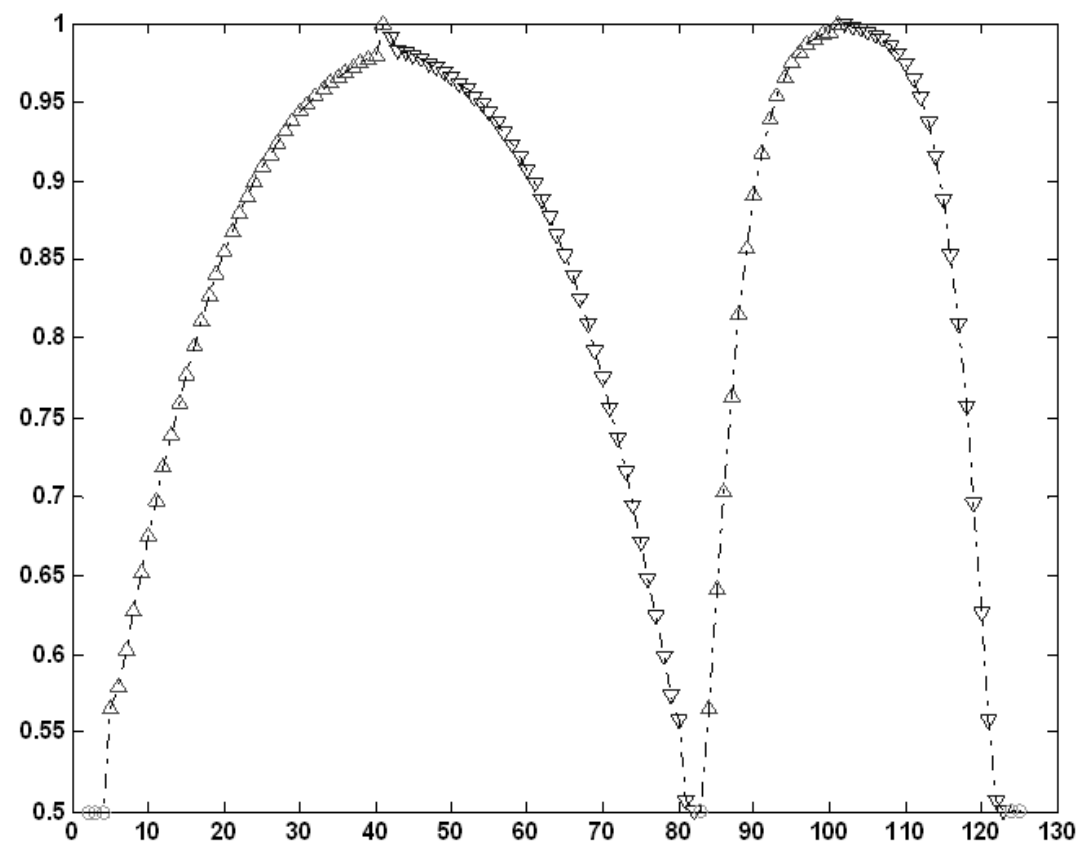

Fig. 4. Output of the new LGMD model on the simulated sequence shown in figure 3. The vertical axis shows the normalized membrane potentials of the LGMD cell; the markers denote the depth movement direction of the object: ' $\triangle$ ' denotes approaching, ' $\nabla$ ' receding and ' $\bigcirc$ ' no significant movement.

both at one pixel per side per frame. It is stationary from frames $79-84$, at size $3 \times 3$, then approaches from $84-101$ and recedes from $101-120$, this time at 2 pixels per edge per frame. It remains stationary again at size $3 \times 3$ for the remainder of the sequence.

Figure 4 shows the output of the LGMD model on the simulated sequence shown in figure 3 . The vertical axis is the normalized membrane potential of the LGMD cell; the marker represents the output of the depth direction cell. This result shows that this model works very well in the simulation dataset.

\subsubsection{Results on real recorded data}

We recorded two short video clips (shown in figures 5 and 7 respectively) for the second experiment, using $320 \times 240$ gray scale images. In these videos (5) a ball is shown, mainly receding to the chair and then bouncing back to approach the camera. There are 18 and 21 frames in the first and second sequences respectively. The first recording has a bigger, fast-moving ball while the second has a smaller, slower-moving ball.

Figure 6 and 8 show the output of the new model on the recorded sequences shown in figure 5 and 7 respectively. In the first dataset, the ball is a bit brighter than background while in the second dataset,the ball is a bit darker than the background. Although the situations are different, the simulation 


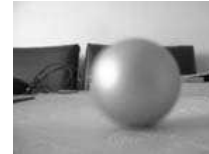

1
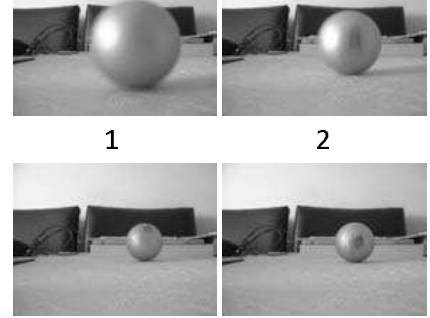

7
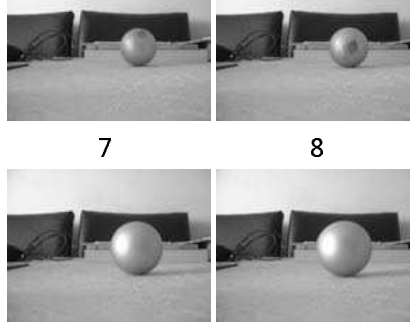

13
2

8

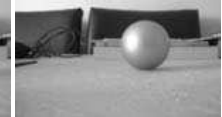

3

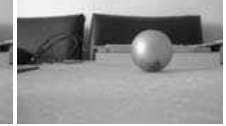

9

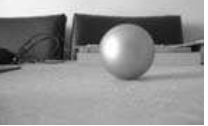

14

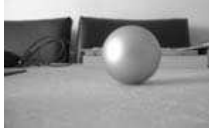

15

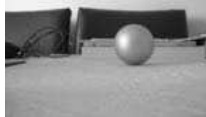

4

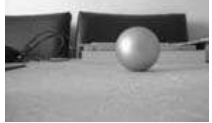

10

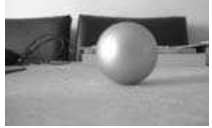

16

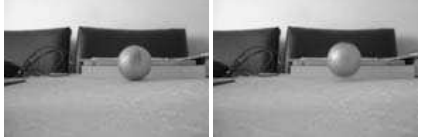

5

6

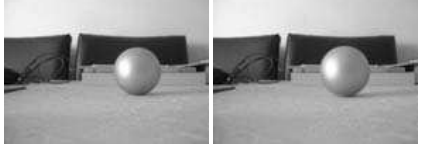

11

12

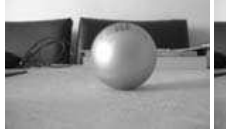

17

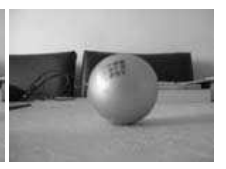

18

Fig. 5. The first recorded sequence. There are 18 frames featuring a ball receding from the camera and then bouncing back to the camera after it hits a chair.

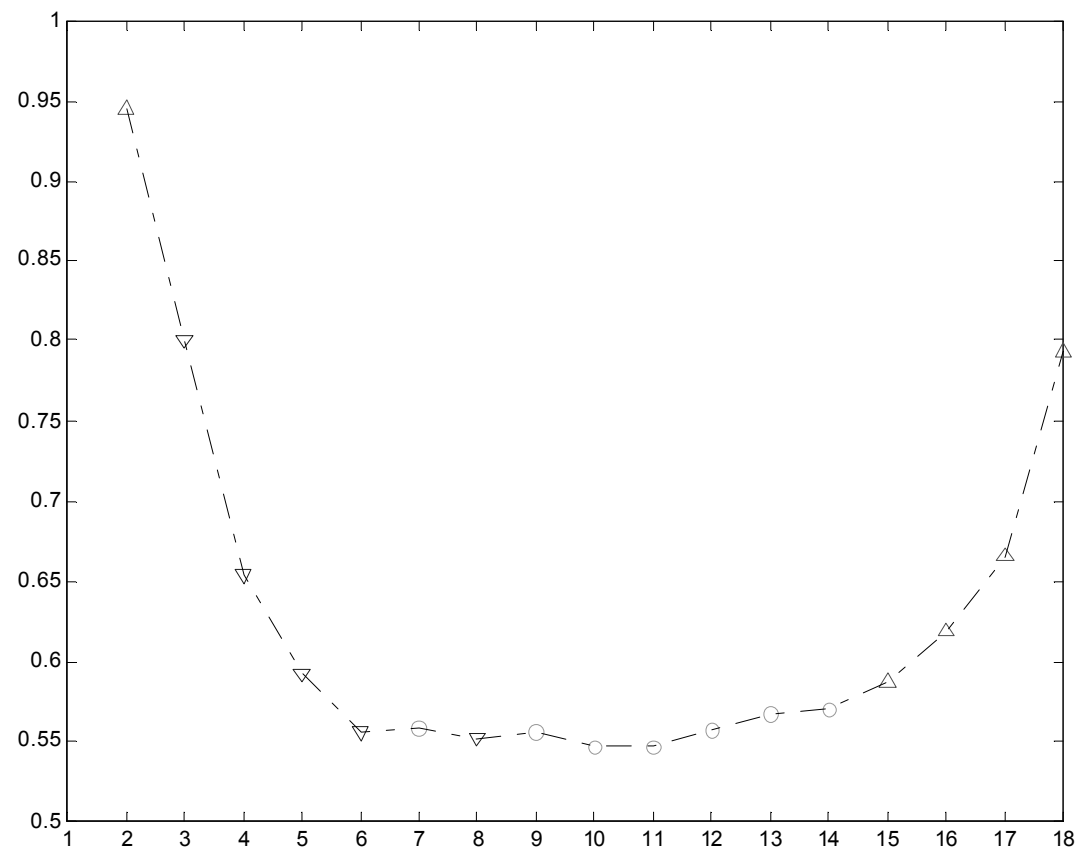

Fig. 6 . The output of the model on the sequence shown in figure 5 . The vertical axis is the normalized membrane potentials of the LGMD cell. The markers denote the depth movement direction; ' $\triangle$ ' denote approaching objects; ' $\nabla$ ' receding objects and ' $\bigcirc$ ' no significant movement.

results are quite similar. We can clearly see that the new model works very well on both recorded data sets. 


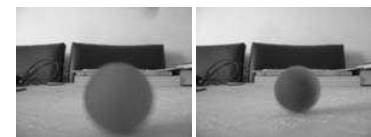

1

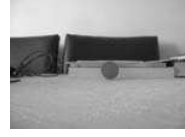

8

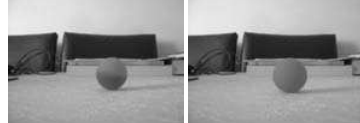

15

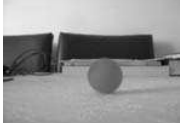

3
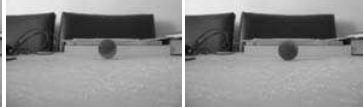

10

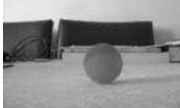

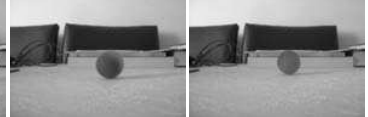

4

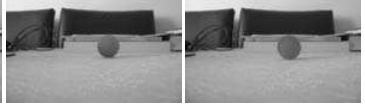

12

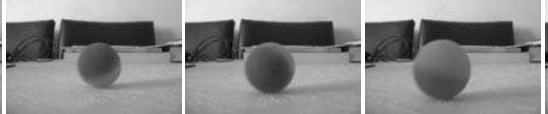

19

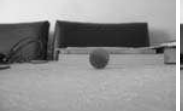

6

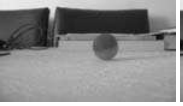

13
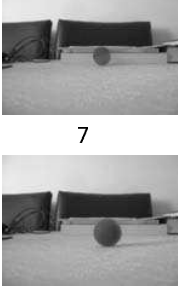

14

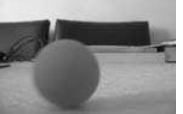

21

Fig. 7. The second recorded sequence. There are 21 frames, featuring a ball receding from the camera and then bouncing back towards the camera after it hits the chair.

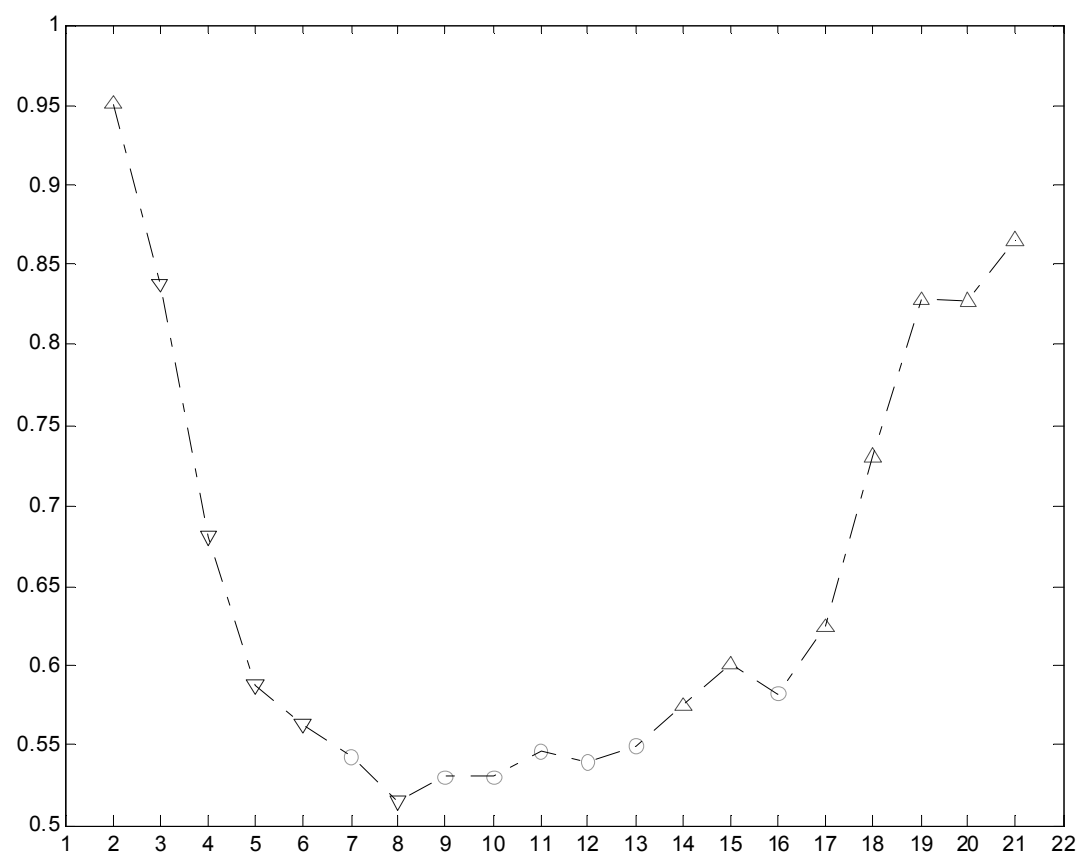

Fig. 8. The output of the model on the sequence shown in figure 7 . The vertical axis is the normalized membrane potentials of the LGMD cell. The markers denote the depth movement direction; ' $\triangle$ ' denote approaching objects; ' $\nabla$ ' receding objects and ' $\bigcirc$ ' no significant movement.

\section{Hardware design and implementation}

The entire collision detection algorithm, based on the modified LGMD as presented in section 3 has been implemented on a Field Programmable Gate Array (FPGA). In contrast to the previously published mixed digital/analogue implementation of the LGMD[35,37], this all-digital implementation has key advantages in easy integration with other digital algorithms on the FPGA. 


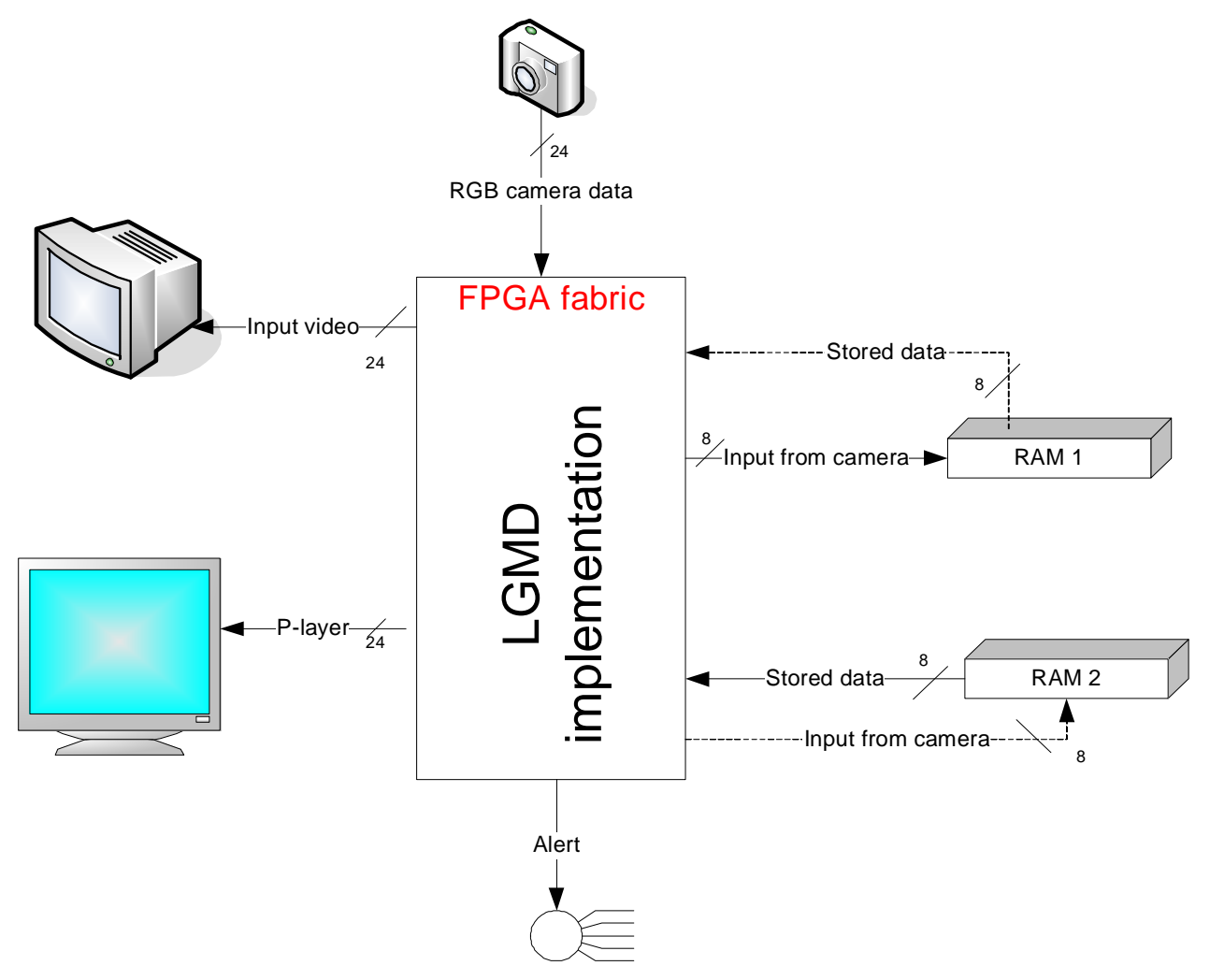

Fig. 9. A high-level block diagram of the FPGA implementation of the modified LGMD model.

\subsection{Overall architecture and platform}

The high-level block diagram of the overall architecture of the system is shown on figure 9. The real-time video stream is input from a digital camera to the FPGA chip, displayed on an monitor and the frames transferred to gray scale images stored in two external RAMs. The neural computing is carried out on the FPGA chip, the excitation S-layer is displayed on another monitor, and an alert is also generated.

Figure 10 shows the system setup. It includes a Celoxica RC340 board, a digital camera and two monitors. The LGMD and $D$ cell outputs are displayed on the board's LCD, and the LEDs (flash lights) are activated on alert. The Celoxica RC340 board is packaged with a Xilinx Virtex-4 XC4VLX160, embedded Block RAM totaling 5,184 Kbits and four banks of ZBT RAM totaling $32 \mathrm{MB}, \mathrm{LCD}$, LEDs and multiple video input and output ports. 


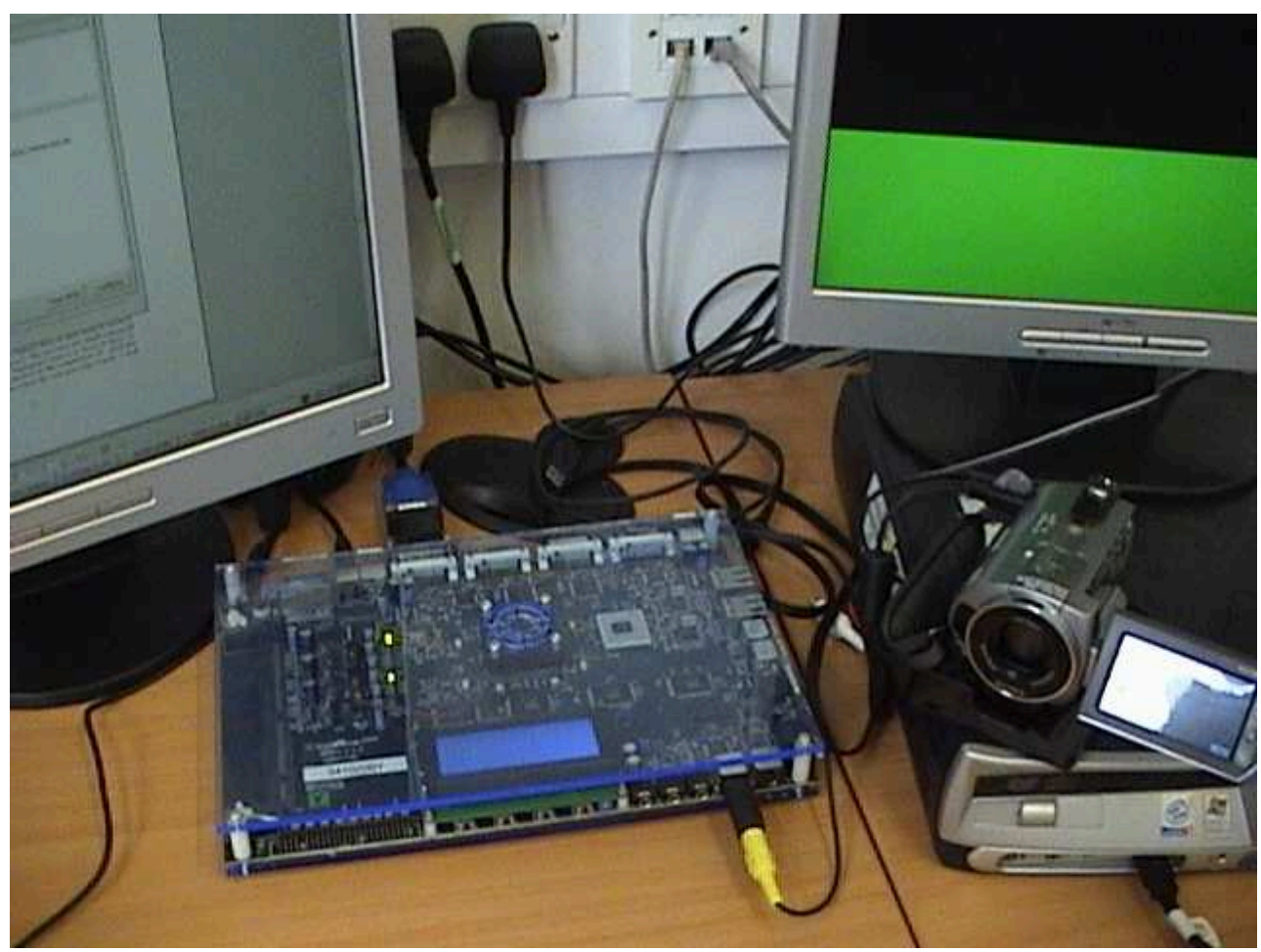

Fig. 10. The system setup includes a Celoxica RC340 board, a digital camera and two monitors. The modified LGMD model lights up the LEDs (flash lights) on the FPGA board based on the values of both LGMD and $D$ cells. These values are also shown on the LCD of the FPGA board.

\subsection{FPGA design}

The FPGA design (see figure 11) has five blocks: the input, P-layer, S-layer, J cell and D cell. The input and P-layer blocks run in parallel, while the S-layer gets triggered when the entire frame has been processed.

The input block reads real-time camera data in 24 bit RGB format and converts it into 8-bit gray-scale intensity. The 8-bit intensity value is written into one of the available RAM blocks whiles the corresponding stored data is read from the other RAM block, serving as the previous pixel value. The 10-bit xlocation and y-location address is also use to address the store data in RAM. The two block of RAM are used to buffer input data from the camera.

The current pixel value (from the camera) and the previous pixel value (from RAM) are used to estimate the luminance P-layer value for the corresponding pixel. This three stage pipeline is completed when an entire frame is captured. The excitatory S-layer is then triggered. This layer uses all eight neighboring pixels in the P-layer. The architecture implemented here is as shown in figure 12. Pixel data from the three rows involved in the computation are copied into a buffer one after the other. The S-layer for each pixel takes exactly three 


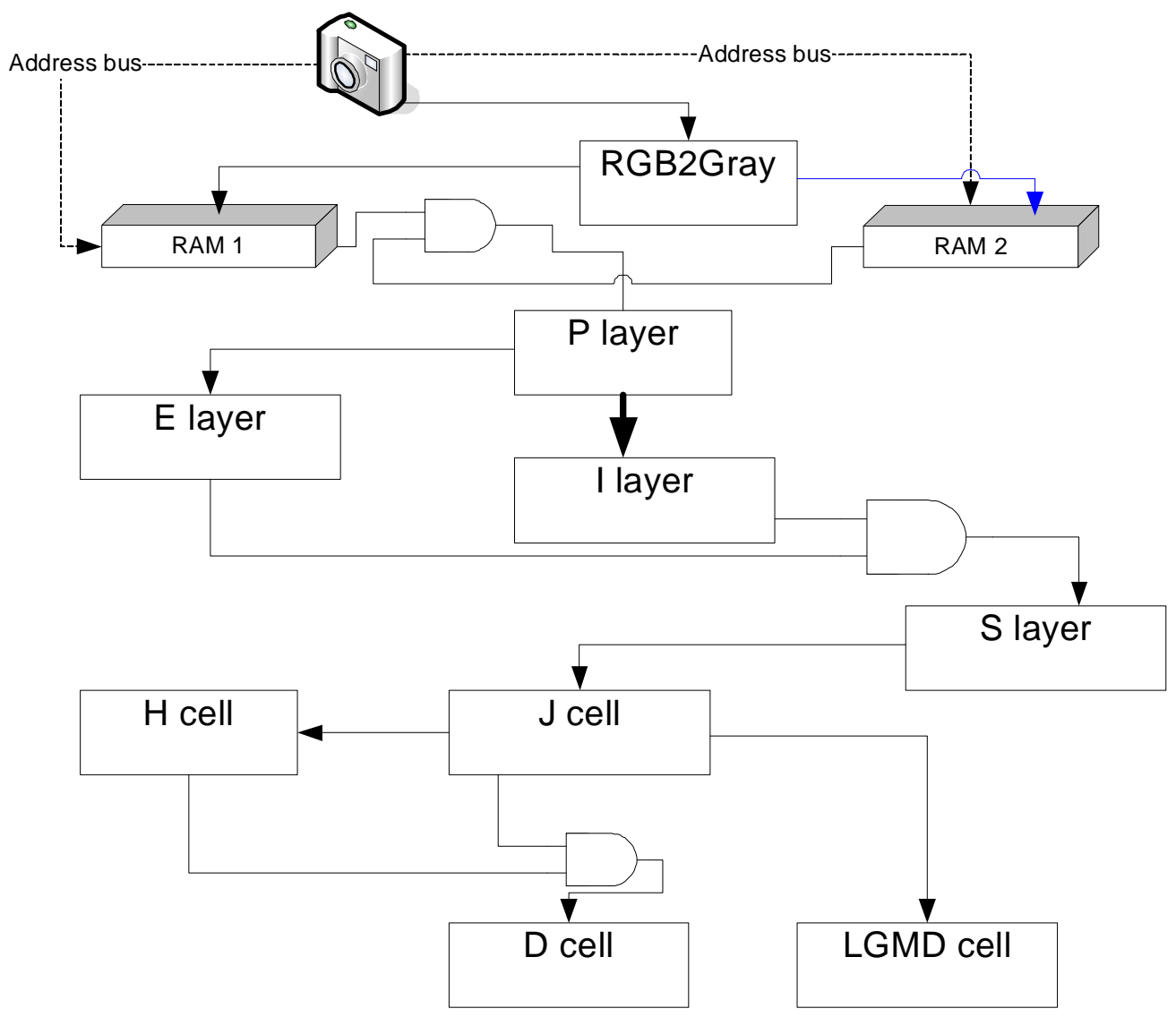

Fig. 11. A high-level circuitry diagram of the various blocks on FPGA

clock cycles, the same number of cycles required to fill the three buffers.

The processing requires seven comparators arranged in a chain as shown in figure 12 and begins execution as soon as the buffer is full. From figure 12, the shaded pixels in the second row are the pixel whose corresponding S-layer value will be generated after three clock cycles.

The S-layer data is passed over to the $\mathrm{J}$ cell, which sums all the pixels values from the S-layer. This block runs in parallel with the S-layer and uses a single accumulator. The $\mathrm{J}$ cell in conjunction with the $\mathrm{H}$ cell is used to generate the value for the $\mathrm{D}$ cell. The $\mathrm{D}$ cell uses the $\mathrm{H}$ cell, which is the delayed $\mathrm{J}$ cell value, as shown in figure 11.

In addition, we simulate equation 11, which determines the output of the $L G M D$ cell from the input, $J$, using a step function, thus avoiding the computation of exponentials and division. We discretize the output, $L G M D$, into the set $\{0.50,0.51, \cdots, 0.99,1.00\}$. Since equation 11 is monotonically increasing in $J$, we can rearrange equation 11 to equation 14, to back-calculate the minimum and maximum values of $J$ that yield a specified value of $L G M D$ (e.g. we plug values of $L G M D=0.505$ and $L G M D=0.515$ into 14 to cal- 


\section{P layer}

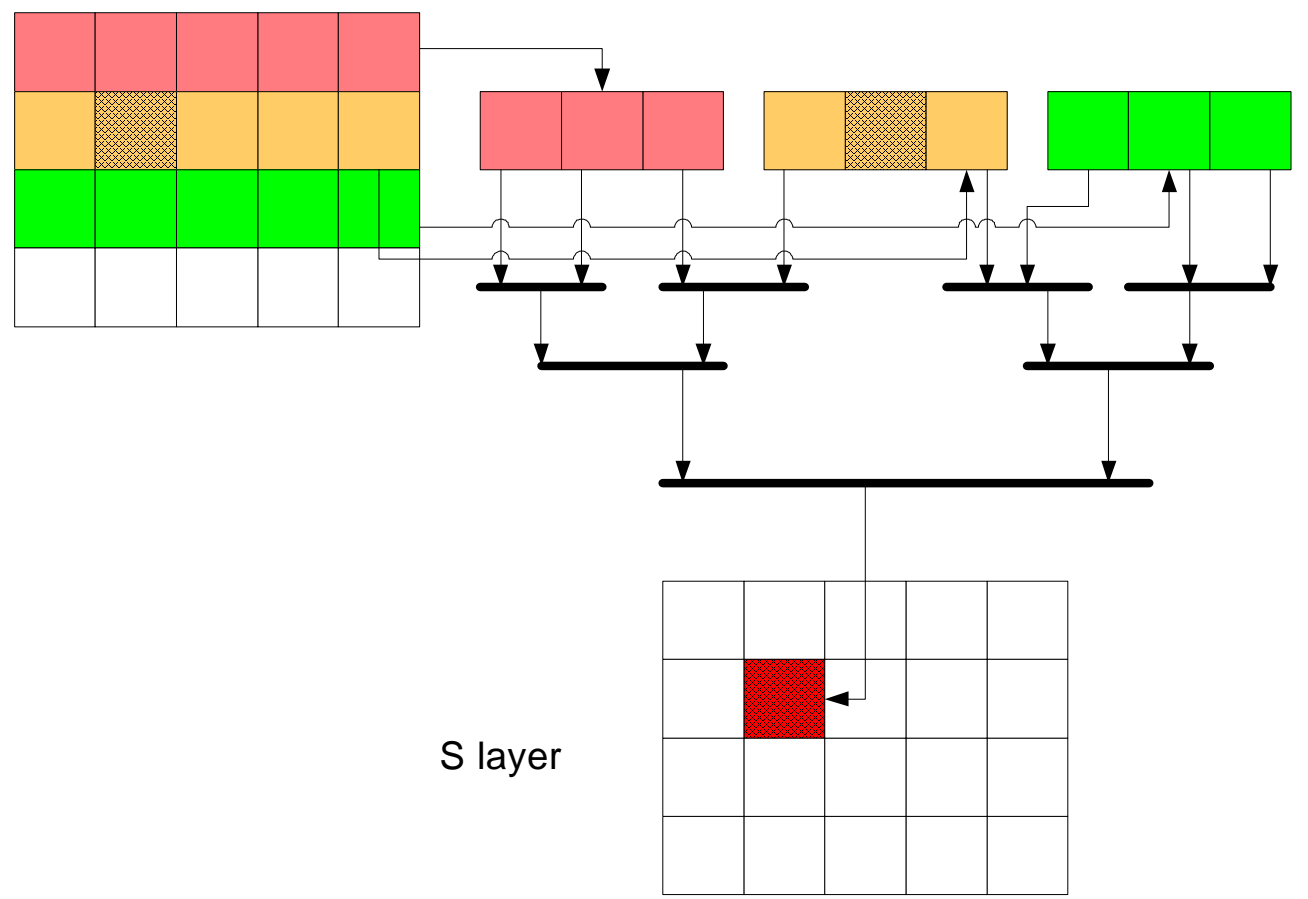

Fig. 12. A detailed translation of the p-layer into s-layer

culate the minimum and maximum values of $J$ that map to $L G M D=0.51$ ). These range limits are checked in parallel to determine the value of $L G M D$ in a single clock cycle.

$$
J_{f}=-\ln \left(L G M D_{f}^{-1}-1\right) \times n_{\text {cell }}
$$

All the layers in the modified LGMD have been implemented on the FPGA fabric with the use of the Block RAM, making it possible to address each layer like a dual-port memory block. The hardware implementation currently excludes the FFI cell as shown in figure 1. However, this can be easily added as it is not computationally complex. The hardware implementation rather makes use of a predefined threshold to estimate the excitation. The excitation of the LGMD cell in figure 12 is very dependent on the value of the D cell; thus if the object is stationary or receding, there is no alert generated at the LGMD cell.

The resources used by the FPGA implementation are listed in table 2. It was implemented on a Xilinx Virtex-4 XC4VLX160 chip, package FF1148 and speed grade -10 . Memory and IO requirements are high, but computational requirements are minimal. 


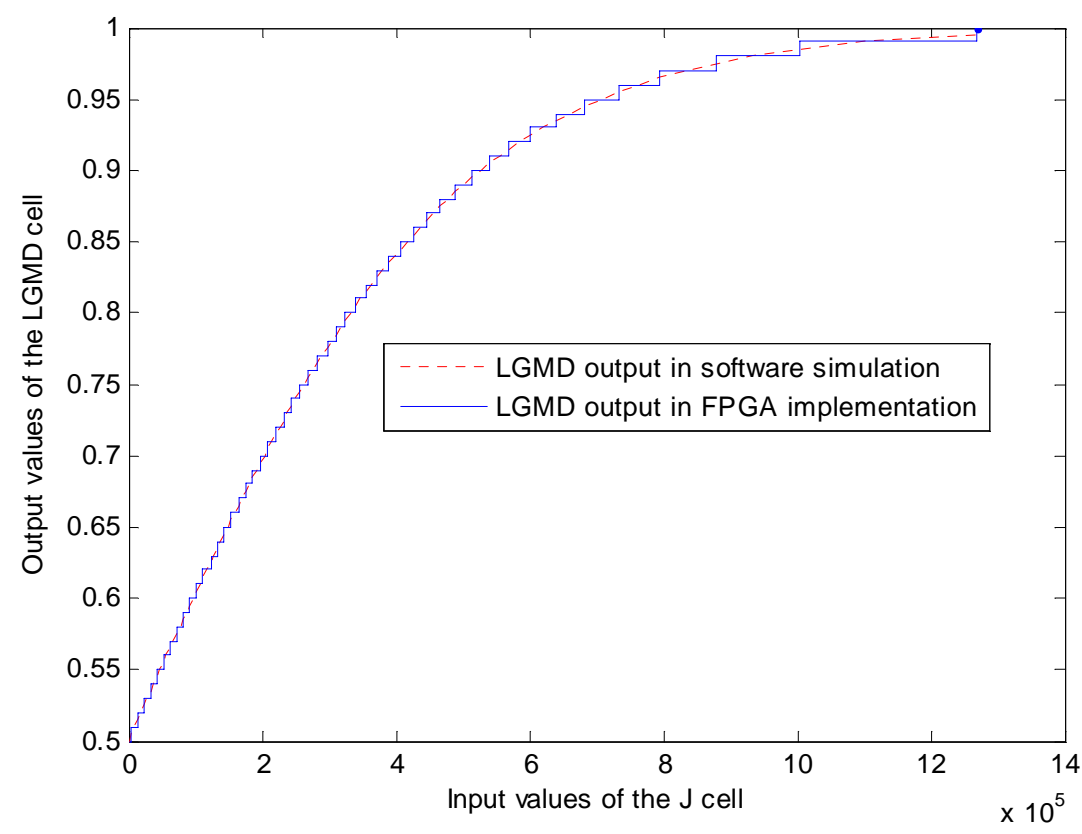

Fig. 13. A step function is used in FPGA implementations for determining the outputs of $L G M D$ cell (vertical axis) from the inputs of $J$ cell (horizontal axis). Only 51 values $\{0.50,0.51, \cdots, 0.99,1.00\}$ were used for the outputs of LGMD cell in the FPGA implementation. Here, the image size is $600 \times 400$.

\section{Table 2}

Implementation results for the modified LGMD, using Virtex-4 $X C 4 V L X 160$, package FF1148 and speed grade -10 .

\begin{tabular}{|l|c|c|c|}
\hline \multicolumn{2}{|c|}{ Resource } & \multicolumn{2}{c|}{ Total Used } \\
\hline Name & Total & Used & Per.(\%) \\
\hline \hline Flip Flops & 135,168 & 2,325 & 1 \\
4 input LUTs & 135,168 & 3,001 & 2 \\
bonded IOBs & 768 & 355 & 46 \\
Occupied Slices & 67,584 & 3,206 & 4 \\
RAM16s & 288 & 285 & 98 \\
\hline
\end{tabular}

\subsection{Hardware testing results}

The hardware implementation has been tested with two frame sizes, $300 \times 200$ and $600 \times 400$. The maximum attainable clock frequency is $50 \mathrm{MHz}$, with $40 \mathrm{MHz}$ being the highest stable frequency. The design takes a total of $3 N+7$ cycles to completely generate an LGMD output, where $N$ is the number of pixels in the entire frame. For frame size $300 \times 200$ running at $40 \mathrm{MHz}$, the system processes approximately 222 frames per second; for frame size $600 \times 400$ the value reduces to 55 frames per second. The low resource utilization of 


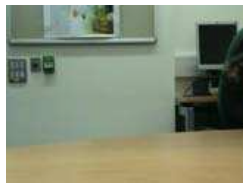

001

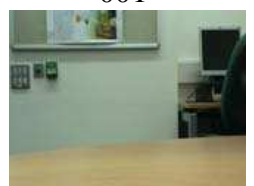

042

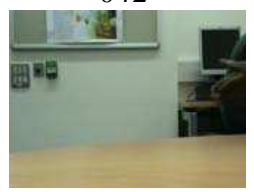

084

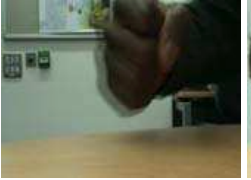

008

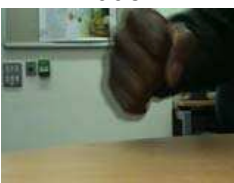

049

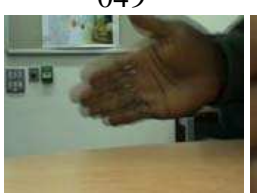

091

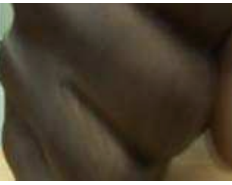

015

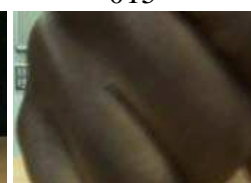

056

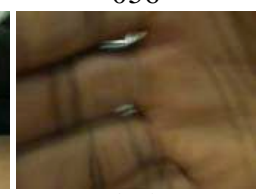

098

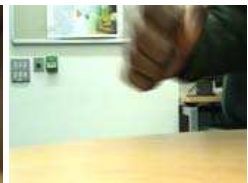

022

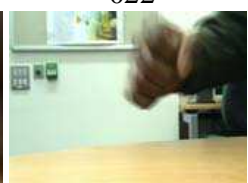

063

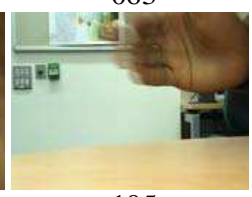

105

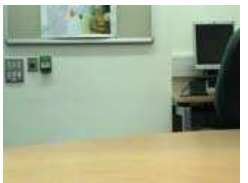

029

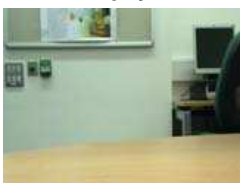

070

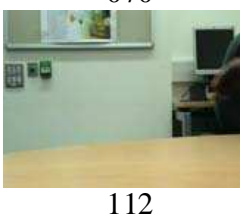

Fig. 14. Frame samples from a video clip of a looming and receding hand movement. The frame numbers are shown under each frame. There are 115 frames, size $600 \times 400$, at frame rate 25 f.p.s.

the implementation makes it possible to run multiple LGMD at the same frequency.

The high computational efficiency makes it possible for the modified LGMD to be used in visual sensor systems with very high frame rate and/or high image resolution.

The reported clock frequency of $40 \mathrm{MHz}$ to $50 \mathrm{MHz}$ also includes the design for controlling the external logic for the 2 VGAs, the camera input and the LEDs for alerts. The design and verification was accomplished using Handel-C high level descriptive language. Compilation and simulation were achieved using the Agility DK design suite. Synthesis, the translation of abstract high-level code into a gate-level netlist, was accomplished using Xilinx ISE tools.

Figure 14 shows a video sequence used to test the hardware implementation. The object (hand) approaches and recedes three times. The video was recorded into the digital camera and the outputs of the LGMD and D cells were written into the external memory, and retrieved for plotting; see figure 15. We can see clearly that the FPGA implementation worked very well in response to this object movement. In comparison with the software simulation results (see figure 6), the curve is not as smooth, due to the step function used in the computation of the LGMD values. Nevertheless, this implementation fulfils the task of giving correct alarms. 


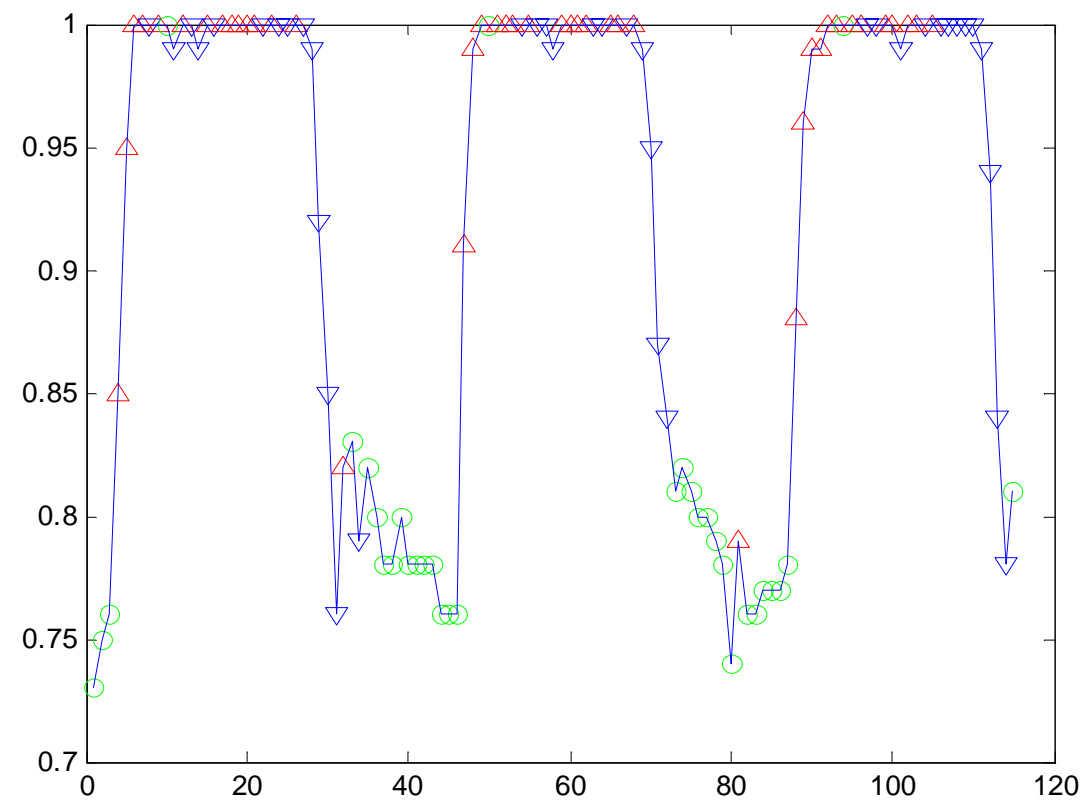

Fig. 15. Experimental results read from external memory of the FPGA board, using the video sequence in figure 14 . The vertical axis is the normalized membrane potentials of the LGMD cell. The markers denote the depth movement direction; ' $\triangle$ ' denote approaching objects; ' $\nabla$ ' receding objects and ' $O$ ' no significant movement.

\section{Conclusion}

In this paper, we propose an LGMD model that provides additional information on the depth direction of the movement. It requires little additional computational cost compared to previous models, and can distinguish approaching from receding objects very quickly.

The new model has been implemented on the Xilinx FPGA chip, and the general purpose design is suitable for transfer to any other FPGA device. The design is compact, occupying limited hardware resources, and therefore be easily integrated with other computational components on a single chip. It has been successfully tested on real-time video clips; experimental results showed hardware performance is consistent with software simulation results.

The high computational efficiency makes the modified LGMD suitable for use in visual sensor systems with very high frame rate and/or high image resolution, and the implementation on a general purpose hardware platform makes it suitable for application in various situations.

In future research we will design a complete chip combining this LGMD model with the specialized translation-sensitive neural network. This will provide both translation and depth movement information, and will work as a general motion tracking sensor. 


\section{Acknowledgments}

The authors would like to thank the UK Trade and Strategy Board for supporting the project under the program grant TP/2/SC/6/I/10444.

\section{References}

[1] F. C. Rind, P. J. Simmons, Seeing what is coming: building collision-sensitive neurones., Trends in Neurosciences 22 (1999) 215-220.

[2] R. D. Santer, P. J. Simmons, F. C. Rind, Gliding behaviour elicited by lateral looming stimuli in flying locusts., Journal of comparative physiology. A, Neuroethology, sensory, neural, and behavioral physiology 191 (1) (2005) $61-73$.

[3] F. C. Rind, R. D. Santer, M. J. Blanchard, P. F. M. J. Verschure, Locust looming detectors for robot sensors, Springer, Berlin, 2003.

[4] S. Judge, F. C. Rind, The locust DCMD, a movement-detecting neurone tightly tuned to collision trajectories, Journal of Experimental Biology 200 (16) (1997) 2209-2216.

[5] F. C. Rind, P. J. Simmons, Orthopteran DCMD neuron: a reevaluation of responses to moving objects. I. selective responses to approaching objects, J Neurophysiol 68 (5) (1992) 1654-1666.

[6] S. B. i Badia, P. F. Verschure, A collision avoidance model based on the lobula giant movement detector neuron of the locust, in: proceedings of IJCNN, 2004, pp. $1757-1761$.

[7] S. Bermudez i Badia, P. Pyk, P. F. Verschure, A fly-locust based neuronal control system applied to an unmanned aerial vehicle: the invertebrate neuronal principles for course stabilization, altitude control and collision avoidance, The International Journal of Robotics Research 26 (7) (2007) 759-772.

[8] F. Rind, D. Bramwell, Neural network based on the input organization of an identified neuron signaling impending collision., Journal of Neurophysiology 75 (1996) 967-985.

[9] M. Blanchard, P. F. M. J. Verschure, F. C. Rind, Using a mobile robot to study locust collision avoidance responses, Int. J. Neural Syst. 9 (5) (1999) 405-410.

[10] M. Blanchard, F. Rind, P. Verschure, Collision avoidance using a model of the locust LGMD neuron., Robotics and Automonous Systems 30 (2000) 17-38.

[11] F. C. Rind, A directionally selective motion-detecting neurone in the brain of the locust: physiological and morphological characterization, Journal of Experimental Biology 149 (1990) 1-19. 
[12] F. C. Rind, Identification of directionally selective motion-detecting neurones in the locust lobula and their synaptic connections with an identified descending neurone, Journal of Experimental Biology 149 (1990) 21-43.

[13] S. Yue, F. C. Rind, Visual motion pattern extraction and fusion for collision detection in complex dynamic scenes, Computer Vision and Image Understanding 104 (1) (2006) 48 - 60.

[14] S. Yue, F. C. Rind, A synthetic vision system using directionally selective motion detectors to recognize collision, Artificial Life 13 (2) (2007) 93-122.

[15] S. Yue, F. C. Rind, Collision detection in complex dynamic scenes using an LGMD-based visual neural network with feature enhancement, IEEE Transactions on Neural Networks 17 (3) (2006) 705-716.

[16] H. Meng, S. Yue, A. Hunter, K. Appiah, M. Hobden, N. Priestley, P. Hobden, C. Pettit, A modified neural network model for lobula giant movement detector with additional depth movement feature, in: Proceedings of IJCNN, USA, 2009, pp. 2078-2083.

[17] C. Connolly, Collision avoidance technology: from parking sensors to unmanned aircraft, Sensor Review 27 (3) (2007) 182-188.

[18] M. Bertozzi, A. Broggi, A. Fascioli, Vislab and the evolution of vision-based UGVs, IEEE Computer 39 (12) (2006) 31-38.

[19] M. Bertozzi, L. Bombini, A. Broggi, P. Zani, P. Cerri, P. Grisleri, P. Medici, Gold: A framework for developing intelligent-vehicle vision applications, IEEE Intelligent Systems 23 (1) (2008) 69-71.

[20] A. Hanazawa, T. Miki, K. Horio (Eds.), Brain-Inspired Information Technology, Vol. 266 of Studies in Computational Intelligence, Springer, 2010.

[21] H. Longuet Higgins, K. Prazdny, The interpretation of a moving retinal image, Proceedings of the Royal Society of London. Series B, Biological Sciences 208 (1980) 385-397.

[22] G. T. Kenyon, Time-to-collision estimation from motion based on primate visual processing, IEEE Trans. Pattern Anal. Mach. Intell. 27 (8) (2005) 1279-1291.

[23] S. Nedevschi, S. Bota, C. Tomiuc, Stereo-based pedestrian detection for collision-avoidance applications, IEEE Trans. Intelligent Transportation Systems 10 (3) (2009) 380-391.

[24] A. Barth, U. Franke, Estimating the driving state of oncoming vehicles from a moving platform using stereo vision, IEEE Trans. Intelligent Transportation Systems 10 (4) (2009) 560-571.

[25] A. Wedel, U. Franke, J. Klappstein, T. Brox, D. Cremers, Realtime depth estimation and obstacle detection from monocular video, in: DAGMSymposium, 2006, pp. 475-484. 
[26] G. Sandini, M. Tistarelli, Active tracking strategy for monocular depth inference over multiple frames, IEEE Trans. Pattern Anal. Mach. Intell. 12 (1) (1990) 1327.

[27] F. Woelk, S. Gehrig, R. Koch, A monocular collision warning system, in: Proceedings of the 2nd Canadian Conference on Computer and Robot Vision, 2005, pp. 220-227.

[28] D. Coombs, M. Herman, T. Hong, M. Nashman, Real-time obstacle avoidance using central flow divergence and peripheral flow, in: IEEE International Conference on Computer Vision, IEEE Computer Society, Los Alamitos, CA, USA, 1995, p. 276.

[29] C. Colombo, Time to collision from first-order spherical image motion, Robotics and Autonomous Systems 31 (1-2) (2000) 5-15.

[30] F. Meyer, Time-to-collision from first-order models of the motion field, IEEE Trans. Robotics and Automation 10 (1994) 792-798.

[31] R. C. Nelson, J. Aloimonos, Obstacle avoidance using flow field divergence, IEEE Trans. Pattern Anal. Mach. Intell. 11 (10) (1989) 1102-1106.

[32] J. L. Barron, D. J. Fleet, S. S. Beauchemin, T. A. Burkitt, Performance of optical flow techniques, International Journal of Computer Vision 12 (1) (1994) $43-77$.

[33] A. Polychronopoulos, M. Tsogas, A. Amditis, L. Andreone, Sensor fusion for predicting vehicles' path for collision avoidance systems, IEEE Transactions on Intelligent Transportation Systems 8 (3) (2007) 549-562.

[34] N. Lazaros, G. C. Sirakoulis, A. Gasteratos, Review of stereo vision algorithms: From software to hardware, International Journal of Optomechatronics 2 (4) (2008) $435-462$.

[35] R. Laviana, L. Carranza, S. Vargas, G. Linan, E. Roca, A bioinspired vision chip architecture for collision detection in automotive applications, in: R. A. Carmona, G. Linan-Cembrano (Eds.), Society of Photo-Optical Instrumentation Engineers (SPIE) Conference Series, Vol. 5839, 2005, pp. 1324.

[36] J. Cuadri, G. Linan, R. Stafford, M. S. Keil, E. Roca, A bioinspired collision detection algorithm for VLSI implementation, Vol. 5839, SPIE, 2005, pp. 238248.

[37] H. Okuno, T. Yagi, Real-time robot vision for collision avoidance inspired by neuronal circuits of insects, in: IROS, 2007, pp. 1302-1307.

[38] H. Okuno, T. Yagi, A visually guided collision warning system with a neuromorphic architecture, Neural Networks 21 (10) (2008) 1431-1438.

[39] R. D. Santer, R. Stafford, F. C. Rind, Retinally-generated saccadic suppression of a locust looming detector neuron: Investigations using a robot locust., Journal of the Royal Society: Interface 1 (2004) 61-77. 
513 [40] S. Yue, F. C. Rind, A collision detection system for a mobile robot inspired by 514 the locust visual system, in: ICRA, 2005, pp. 3832-3837.

515 [41] S. Yue, F. C. Rind, M. S. Keil, J. Cuadri, R. Stafford, A bio-inspired visual collision detection mechanism for cars: Optimisation of a model of a locust neuron to a novel environment, Neurocomputing 69 (13-15) (2006) 1591-1598. 